\title{
Dielectric properties of nonaqueous electrolyte solutions
}

\author{
J. Barthel and R. Buchner \\ Institut für Physikalische und Theoretische Chemie der Uni- \\ versität Regensburg, D- 8400 Regensburg, Federal Republic of \\ Germany
}

\begin{abstract}
High frequency permittivity of non-aqueous electrolyte solutions and their solvents is discussed in the framework of time domain and frequency domain methods. Evaluation of cata is performed on the basis of models presupposing one or more relaxation processes. Depression of the solvent permittivity, relaxation times of the solvent molecules, and dispersion amplitudes and relaxation times of ion-pair relaxation are investigated with regard to actual theories underlying these phenomena. Information is obtained on structural and dynamical properties of classes of electrolyte solutions based on protic H-bonding, dipolar aprotic, aná low permittivity solvents and mixtures of solvents.
\end{abstract}

\section{INTRODUCTION}

During the last decade new technologies have attracted the interest of chemical engineers in non-aqueous solutions, intensively studied nowadays because of their wide range of physical properties. Up to now the dielectric properties of these ion conducting systems have been almost unexamined, despite their fundamental importance. Chemists are keen to obtain information on the structure of solutions which can be provided by permittivity measurements. Theoreticians hope to get more insight into the dynamic and transport properties of electrolyte solutions from a knowledge of relaxation times anc aielectric decrements.

Dielectric data of electrolyte solutions result from the application of electromagnetic fields to the systems investigated. The phenomenological description of the interaction between electromagnetic fields and material systems is based on a set of equations composed of four differential equations

$$
\text { rot } \overrightarrow{\mathrm{H}}=\overrightarrow{\mathrm{I}}+\partial \overrightarrow{\mathrm{D}} / \partial t ; \quad \text { rot } \overrightarrow{\mathrm{E}}=-\partial \overrightarrow{\mathrm{B}} / \partial t ; \operatorname{div} \overrightarrow{\mathrm{D}}=\rho ; \operatorname{div} \overrightarrow{\mathrm{B}}=0 \quad(1 \mathrm{a}-\mathrm{a})
$$

and three material equations

$$
\vec{D}=\varepsilon_{0} \varepsilon \vec{E} ; \vec{I}=K \vec{E} ; \quad \vec{B}=\mu_{0} \mu \vec{H}
$$

Equations (1a) and (1b) are the liaxwell equations; ecjuations (1c) and (1ci) are the Doisson equations of the electric and nagnetic field; the material equations link the electric vectors $\vec{D}$ (electric displacement) and $\vec{E}$ (electric field strength), the magnetic vectors $\vec{B}$ (magnetic flux density) and $\vec{H}$ (magnetic field strength), and the electric current density $\vec{I}$ and field strength $\vec{E}$ of conducting systems with the help of material properties $\varepsilon$ (relative permittivity), $\mu$ (relative permeability) and $k$ (specific electric conductance); $\varepsilon$ is the permittivity of vacuum $\left(\varepsilon_{0}=\left(\mu_{c} c^{2}\right)^{-1}\right) ; \mu_{0}$ is the permeability of vacuum, $c$ is the speed of light; $\rho$ is the charge density of the electric field.

Starting from a phenomenological description, the response of an electrolyte solution to a varying electromagnetic field can be separated in an ohmic current density $\vec{I}$ of specific conductance $\kappa$ and a displacement current aensity $\partial \overrightarrow{\mathrm{D}} / \partial t$ measured by the relative permittivity $\varepsilon$ (eris. (1a), (1e) and (1f)); the relative permeability $\mu$ of the solution is not involved. The ohrnic contribution is a consequence of charge transport by the movement of the free ions whereas the displacement current contribution originates from the macroscopic dipole moment of the solution characterized in the phenomenological description of dielectric properties with the help of the dielectric polarization $\vec{P}$ (dipole moment of the solution per unity of volume). 
TABLE 1. Recent studies on high frequency permittivities of protic and aprotic solvents and their electrolyte solutions showing no ionic relaxation processes

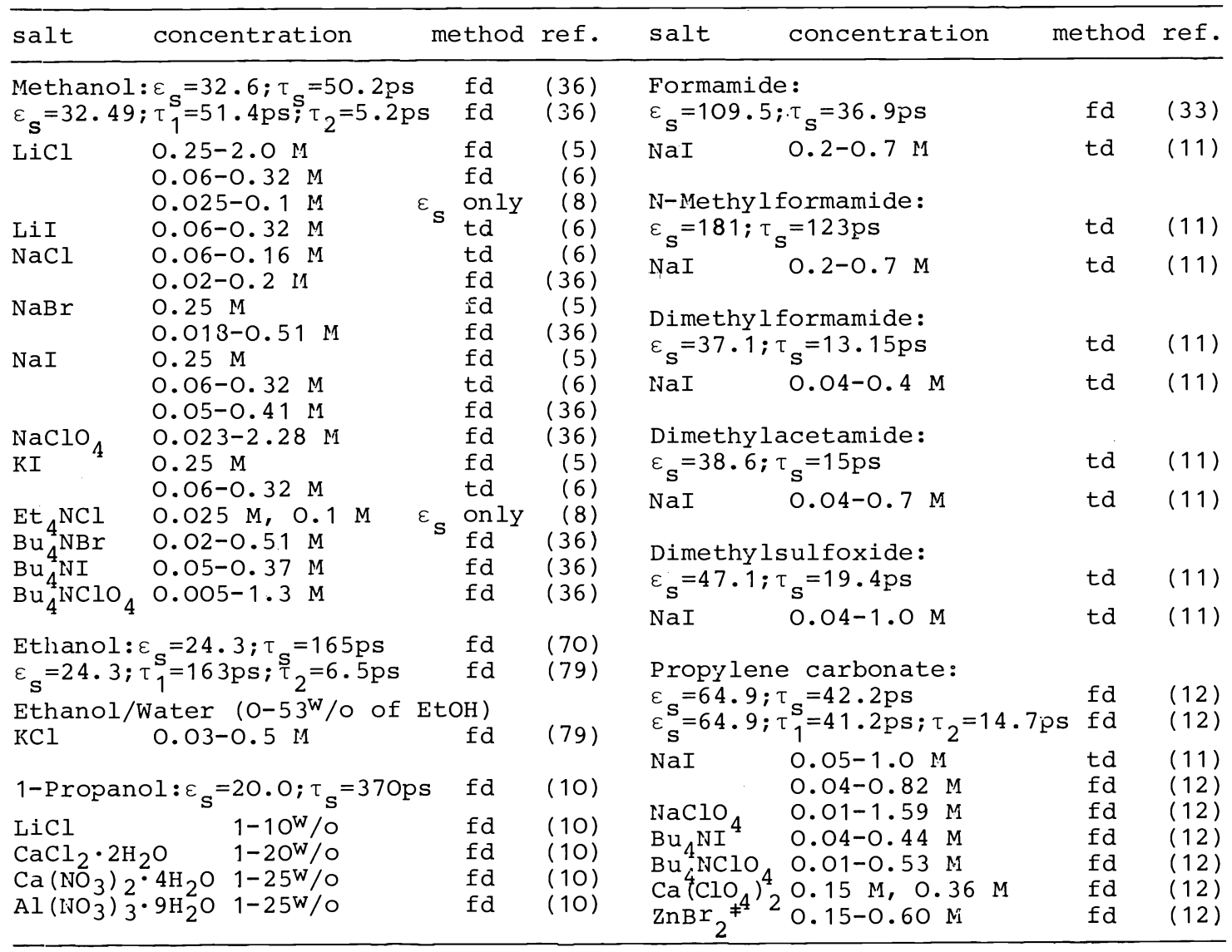

TABLE 2. Recent studies on high frequency permittivities of electrolyte solutions showing ionic relaxation processes

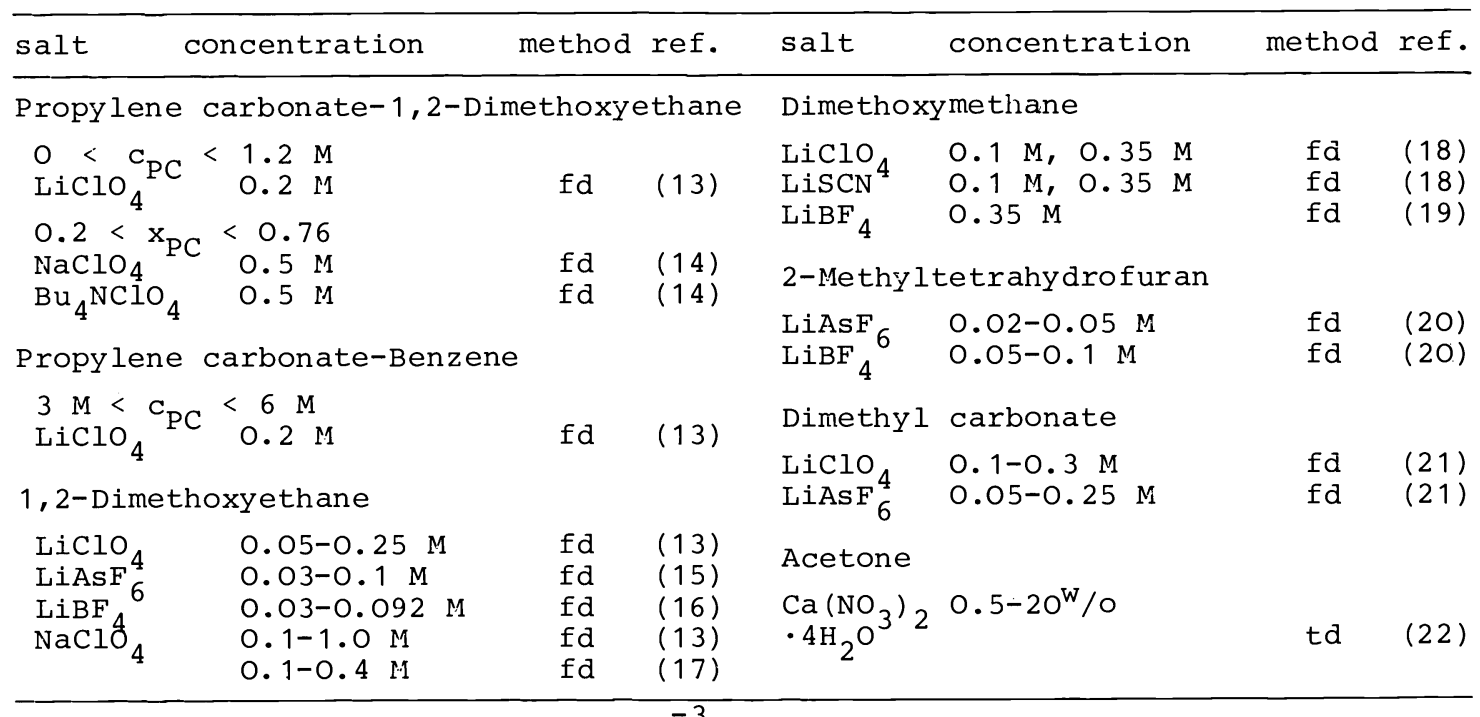

LEGEND to TABLES 1 and $2 . \mathrm{M}$, mol $\mathrm{dm}^{-3} ; \mathrm{w} / \mathrm{o}$, weight per cent; $\mathrm{x}_{\mathrm{PC}}$ or c ce, mole fraction or concentration (mol $\mathrm{dm}^{-3}$ ) of propylene carbonate; $\varepsilon_{\mathrm{S}}$, relative permittivity of the pure solvent; $\tau_{s}=\tau_{1}$, main relaxation time of the pure solvent; $\tau_{2}$, high frequency relaxation time of the pure solvent; methods: fd, frequency domain; td, time domain; $\neq_{\text {with }}$ ionic relaxation process. 


$$
\overrightarrow{\mathrm{P}}=\overrightarrow{\mathrm{D}}-\varepsilon_{\mathrm{O}} \overrightarrow{\mathrm{E}}
$$

According to eq. (1a) only the sum of the conductance and permittivity effects contributing to the response of the electrolyte solution can be experimentalli observed; assumptions must be made to enable separate discussions of these

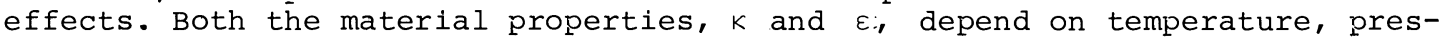
sure, composition of the solution, and frequency. The commonly used assumption is to consider specific conductance as a frequency indepenỏent quantity and to equate it to its static value, cf. ref. (1).

Dielectric polarization of an electrolyte solution results mainly from the permanent and induced dipole moment of the solvent molecules. Permittivity studies thus can probe solvent-solvent and ion-solvent interactions and yield information complementary to studies of thermodynamic or transport properties on electrolyte solutions; in some cases ionic contributions to the electric moment can be detected which are the conseciuence of ion association into pairs and higher aggregates. Unique results are obtained from measurements at frequencies in the dispersion region of permittivity which yield relaxation times characteristic of structural rearrangements and which are essentially inaccessible by other methods.

Despite these possibilities investigations have been few in number on dielectric properties of electrolyte solutions, especially the non-aqueous electrolyte solutions, which are the subject of this paper. Compilations of the literature to 1975 can be found in articles and monographs (1-4); more recent publications are quoted in Tables 1 and 2 .

The reasons for the lack of information are the experimental problems: precise measurements at low electrolyte concentrations (< $0.1 \mathrm{~mol} \mathrm{dm}^{-3}$ ), expensive equipment, and a lack of theoretical concepts which in turn require a knowledge of permittivity data at very low concentrations. There is hope to escape from this vicious circle through high frequency measurements carried out at low concentrations (about 0.005 mol $\mathrm{dm}^{-3}$ ) with new precise measuring equipment. Some of the results of this work are presented in this paper.

\section{EXPERIMENTAL METHODS}

\section{Time-domain and frequency-domain methods}

To begin with, a non-conducting system is considered. The application of a static field $\mathrm{E}$ produces an equilibrium polarization $\langle\overrightarrow{\mathrm{P}} \mathrm{ec}\rangle$ as the response.

$$
\left\langle\overrightarrow{\mathrm{P}}^{\mathrm{eq}}\right\rangle=\varepsilon_{0}(\varepsilon-1) \quad \overrightarrow{\mathrm{E}}=\left\langle\overrightarrow{\mathrm{P}}_{\mu}\right\rangle+\left\langle\overrightarrow{\mathrm{P}}_{\alpha}\right\rangle
$$

In eq. (3) $\langle\overrightarrow{\mathrm{P}}>$ results from the alignement of the molecular permanent moments $\vec{\mu}$ against thermal motion; $\langle\overrightarrow{\mathrm{P}} \alpha\rangle$ is due to the induced moments $\vec{\mu}_{\text {ind }}\left(\vec{\mu}_{\text {in }}=\alpha \vec{\alpha}^{\prime}\right)$ which result from the polarizability $\alpha$ of the molecules.

The application of high freciuency fields $\vec{E}$ entails dielectric dispersion, as a consequence of the finite time needed, to build up a new equilibrium value of $\langle\overrightarrow{\mathrm{P}}\rangle$. Dispersion is always coupled with energy absorption. Inducea polarization $\left\langle\overrightarrow{\mathrm{P}}{ }_{\alpha}>\right.$ fluctuates very fast leading to the well-known absorption spectra in the $I R$ and UV regions, whereas the relaxation time of $\left\langle\overrightarrow{\mathrm{P}} \mu_{\mathrm{p}}\right\rangle$, in polar solvents characteristic for molecular reorientation, is normally in the pico- and nanosecond range.

Two experimental techniques are distinguished, the time-domain and frequencydomain methods.

A field jump at time $t_{0}, t_{0}=0$ in Fig. 1a, yields a step-response function of polarization $\mathrm{F}_{\mathrm{P}}(\mathrm{t})$ (1)

$$
F_{P}(t)=\langle\vec{P}(0) \cdot \vec{P}(t)\rangle /\langle\vec{P}(0) \cdot \vec{P}(0)\rangle
$$

The step-response function of the orientational polarization $\mathrm{F}_{\mathrm{P}}^{\mu}$ is of the relaxational type, i.e. monotonously decreasing with time, see Fig. $1 \mathrm{a}$.

Application of a harmonically varying field, $\vec{E}=\vec{E}_{0} e^{i \omega t}$, of sufficiently high frequency (in the microwave region of about $100 \mathrm{MHz}$ to $300 \mathrm{GHz}$ ) will result in a phase shift $\delta$ of polarization against $\overrightarrow{\mathrm{E}}(t), \mathrm{Fig} .1 \mathrm{~b}$, and an amplitude reduction with regard to static $\langle\vec{p} e q\rangle$. The amplitude reduction is expressed through the real part $\varepsilon^{\prime}(\omega)$ of the complex permittivity $\hat{\varepsilon}(\omega)$ 


$$
\hat{\varepsilon}(\omega)=\varepsilon^{\prime}(\omega)-i \varepsilon "(\omega)
$$

where $\omega, \omega=2 \pi \nu$, is the circular and $\nu$ the linear frequency of the electromagnetic wave. The loss factor $\varepsilon^{\prime \prime}(\omega)$ measures the energy dissipation ( $\left.\frac{1}{2} \mathrm{E}_{0}^{2} \omega \varepsilon_{0} \varepsilon^{\prime \prime}\right)$ in the solution, and is related to the phase shift $\delta\left(\tan \delta=\varepsilon^{\prime \prime} / \varepsilon^{\prime}\right)$.

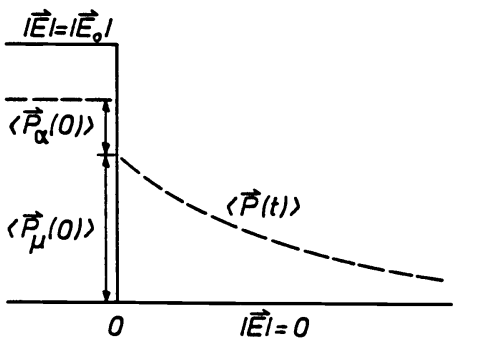

(a)

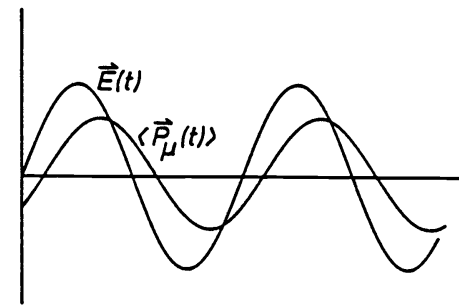

(b)

Fig. 1. Methods for the determination of complex permittivity. (a) time domain method: Application of a jump in the field strength $|\mathrm{E}|$ and measurement of the response $\langle\overrightarrow{\mathrm{P}}(t)\rangle=\langle\overrightarrow{\mathrm{P}}(0)\rangle \mathrm{F}_{\mathrm{P}}(t)$ (b) frequency domain method: Application of a harmonic field $\vec{E}(t)=\vec{E}_{0} \exp (i \omega t)$ and measurement of amplitude and phase of the response $<\overrightarrow{\mathrm{P}}_{\mu}(t)>=\varepsilon_{0}\left(\hat{\varepsilon}(\omega)-\varepsilon_{\infty}\right) \overrightarrow{\mathrm{E}}(t)$

Time-domain measurements determining the step-response function and frequencydomain experiments yielding complex permittivities are linked, c6. refs. $(1,9)$, via the relationship

$$
\hat{\varepsilon}(\omega)=\varepsilon_{\infty}+\left(\varepsilon-\varepsilon_{\infty}\right) \int_{0}^{\infty}\left[-d F_{P}^{\mu}\left(t^{\prime}\right) / d t^{\prime}\right] e^{-i \omega t^{\prime}} d t^{\prime}
$$

In eq. (6) $\varepsilon_{\infty}$ is the permittivity of the sample when only induced moments contribute to polarizability.

A variety of methods, for both the time-domain and the frequency-domain, have been developed for the experimental determination of complex permittivity data of liquids in the microwave region. Detailed information of these is given in references $(23-28)$.

The major advantage of the time-domain techniques is the possibility of probing the dielectric properties of a sample in the frequency range between several $\mathrm{MHz}$ and $10 \mathrm{GHz}$ within a few minutes. If the conductance is not too high (10, 11,29 ) time-domain measurements now seem to be competitive in accuracy (about $5 \%$ in $\varepsilon^{\prime}$ and $\varepsilon^{\prime \prime}$ ) with frequency-domain methods up to about 3-4 GHz.

From 1 to $100 \mathrm{GHz}$ frequency-domain techniques using the method of travelling waves introduced by Buchanan (30) yield superior results. In this method an electromagnetic wave transmitted by the sample is probed interferometrically. However, these measurements are expensive and time-consuming owing to the unavoidable use of narrow band wave guide devices.

For conducting systems like electrolyte solutions the same techniques can, in principle, be used. Experimental problems are greater because of the additional conductivity loss and the response of the solute/electrode interface at low frequencies (2). Some methods giving precise results for low loss liquids cannot be used, e.g., cavity resonators.

As already stressed only a generalized permittivity $\hat{\varepsilon}_{\text {tot }}(\omega)$ can be measured. This is the sum of the contributions from ohmic and displacement current contributions.

$\hat{\varepsilon}_{\text {tot }}(\omega)=\hat{\varepsilon}(\omega)+\hat{\kappa}(\omega) /\left(i \omega \varepsilon_{0}\right) ; \varepsilon_{\text {tot }}^{\prime}(\omega)=\varepsilon^{\prime}(\omega) ; \varepsilon_{\text {tot }}^{\prime \prime}(\omega)=\varepsilon^{\prime \prime}(\omega)-\kappa /\left(\omega \varepsilon_{0}\right) \quad(7 a, b, c)$

Eq. (7a) is obtained by combining eqs. (1a), (1e) and (1f); eqs. (7b) and (7c) result from the assumption of frequency independent specific conductance.

\section{Method of travelling waves}

In our laboratory five experimental arrangements of the travelling-waves method are used covering the range from 0.95 to $40 \mathrm{GHz}$. Details of this 
equipment are given in refs. $(3,36)$ based on the 'know-how' acquired with preceding apparatus (31-34). Purification of solvents, preparation of solutions, and measurements are carried out under a positive pressure of solvent saturated nitrogen gas such that the solutions are screened against evaporation and are never exposed to the atmosphere $(3,4,36)$.

The real and imaginary parts of permittivity at frequency $v$ are obtained from the measurement of the nedium wave length $\lambda_{m}$ and the attenuation exponent $\alpha$ of the samples

$$
\varepsilon^{\prime}(\omega)=\left(\frac{c}{v \lambda_{m}}\right)^{2}-\left(\frac{c \alpha}{2 \pi \nu}\right)^{2}+\left(\frac{c k}{2 \pi \nu}\right)^{2} ; \varepsilon_{\text {tot }}^{\prime \prime}(\omega)=\left(\frac{\alpha}{\pi \lambda_{m}}\right)\left(\frac{c}{v}\right)^{2}
$$

In eqs. (8) $\mathrm{c}$ is the speed of light, and $\mathrm{k}_{\mathrm{c}}$ is the cut-off wave number of the wave guide in the measuring cell.

With this equipment precision is better than $0.4 \%$ for $\varepsilon^{\prime}(\omega)$ and $0.3 \%$ for $\varepsilon "(\omega)$ at all frequencies; reliable standards are lacking for a good assessment of accuracy.

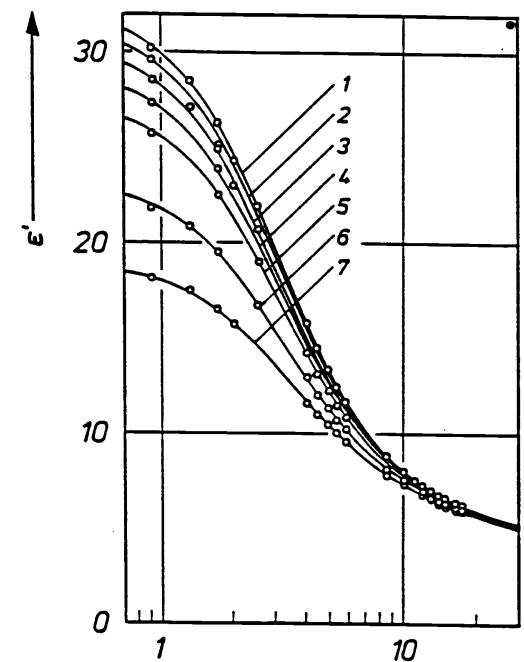

(a)

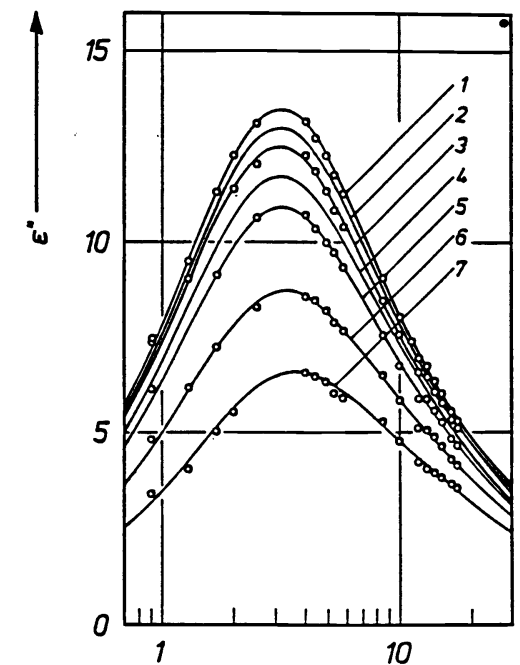

(b)

Fig. 2. Dispersion (a) and absorption (b) curves of methanol solutions of $\mathrm{NaClO}_{4}$ at $25^{\circ} \mathrm{C}(36): 1$, pure methanol; 2, $0.02 \mathrm{~N}$; 3, $0.05 \mathrm{M} ; 4,0.10 \mathrm{Ni} ; 5,0.19 \mathrm{M} ; 6,0.42 \mathrm{M} ; 7,0.84 \mathrm{M}$.

As a typical example dispersion and absorption data of methanol solutions of $\mathrm{iNaClO}_{4}$ are given in Figs. 2 .

\section{Data analysis}

Nowadays computer facilities are readily available and curve fitting is usually done numerically; for special problems see refs. $(2,37)$.

A comprehensive study in our laboratory on using the different dispersion equations, Debye (38), Cole-Cole (39), Davidson-Cole (40), Havriliak-Negami (41), Fröhlich (42), and Fuoss-Kirkwood combined with the Kramers-Kronig relationship (78) yield the result that the assumption of a single relaxation process, $i . e .$, a Debye equation with a single relaxation time generally does not reproduce high frequency permittivitites within frequency regions large enough for the reliable interpretation of physical effects.

Superposition of relaxation processes is assurned when more than one molecular process is involved in dispersion. According to Debye's model the dispersion of permittivity $\varepsilon(\omega)$, for $N$ relaxation processes with iv relaxation times $\tau_{j}$ and $\mathrm{N}$ weight factors $\mathrm{g}_{j}$ is given $b_{I}$ the relationships

$$
\varepsilon(\omega)=\varepsilon_{\infty}+\left(\varepsilon_{1}-\varepsilon_{\infty}\right) \sum_{j=1}^{N} \frac{g_{j}}{1+i \omega \tau_{j}} ; g_{j}=\frac{\varepsilon_{j}-\varepsilon_{j \infty}}{\varepsilon_{1}-\varepsilon_{\infty}} ; \sum_{j=1}^{N} g_{j}=1
$$

In eq. (9a) $\varepsilon_{1}$ and $\varepsilon_{\infty}$ are the permittivities of the solution at zero and infinite frequencies, $i . e$. the upper $(\nu \rightarrow 0)$ and lower $(\nu \rightarrow \infty)$ limit of permittivit $\ddot{y}$. 
The weight factor $g_{j}$ of the $j-t h$ relaxation region, eq. (9b), is given by the ratio of its partial $\left(\varepsilon_{j}-\varepsilon_{j \infty}\right)$ and the total dispersion amplitude $\left(\varepsilon_{1}-\varepsilon_{\infty}\right)$. The over-all relaxation curve is a seciuence of semicircles of radius $\left(\varepsilon_{j}-\varepsilon_{j \infty}\right) / 2$ having in cormon the point $\varepsilon_{j-1, \infty}=\varepsilon_{j}$ on the $\varepsilon^{\prime}$-axis, see eq. $(9 \mathrm{c}) ; \varepsilon_{\mathrm{N}_{\infty}}=\varepsilon_{\infty}$. Fig. 3 shows an example with three relaxation regions.

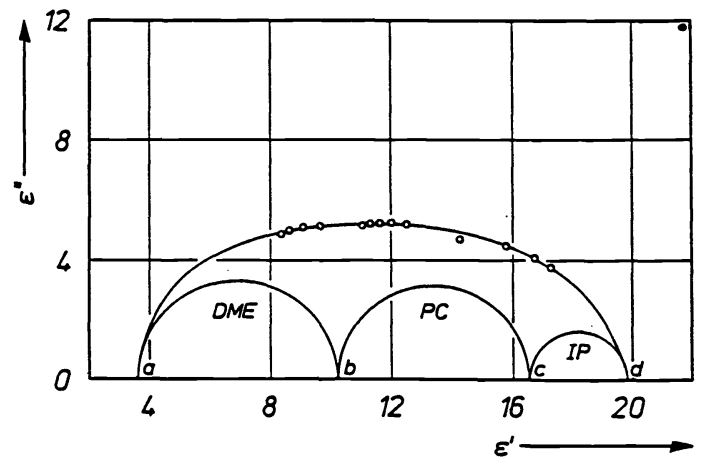

Fig. 3. Cole-Cole plot of the system propylene carbonate $\left(\mathrm{x}_{\mathrm{PC}}=0.20\right)-1.2$-dimethoxyethane-0.48 $\mathrm{M} \mathrm{NaClO}_{4}$ at $25^{\circ} \mathrm{C}(14)$. a , $\varepsilon_{\infty}=\varepsilon_{3 \infty}=$ $\varepsilon_{\infty}(\mathrm{DME}) ; \mathrm{b}, \varepsilon_{3} \stackrel{\infty}{=} \varepsilon_{\mathrm{S}}\left(\mathrm{DMM}^{\infty}\right)=$ $\left.\varepsilon_{\infty}(\mathrm{PC}) ; \mathrm{c} ; \varepsilon_{2}=\varepsilon_{\mathrm{S}} \mathrm{PPC}\right)=$ $\varepsilon_{\infty}^{\infty}(\mathrm{IP}) ; \mathrm{d}, \varepsilon_{1}=\varepsilon(\mathrm{IP})$; $\tau_{3}=\tau(\mathrm{DME})=9.8 \mathrm{DS} ; \tau_{2}=$ $\tau(\mathrm{PC})=49 \mathrm{ps} ; \tau_{1}=\tau(\mathrm{IP})=$ 203 ps

If the relaxation times $\tau_{j}$ of a sequence of processes show only small differences cole-Cole plots, or other previously ciuoted methods may be successfully used for the representation of the characteristic dispersion and spectral functions of the relaxation time distribution; for exarnles see refs. (12,43, 44 ).

In our laboratory the appropriate parameters, $e . g ., \varepsilon_{j}, \varepsilon_{j \infty}$ and $\tau_{j}$ of Debye plots or the parameters of Cole-Cole plots, are evaluated with the help of CURFIT (77) programs which find the minimum of the function

$$
\chi^{2}=\frac{1}{2 m-n} \sum_{i=1}^{m}\left[\left(\frac{\varepsilon_{i}^{\prime}-\varepsilon_{i, c a l c}^{\prime}}{\sigma\left(\varepsilon_{i}^{\prime}\right)}\right)^{2}+\left(\frac{\varepsilon_{i}^{\prime \prime}-\varepsilon_{i, \text { calc }}^{\prime \prime}}{\sigma\left(\varepsilon_{i}^{\prime \prime}\right)}\right)^{2}\right],
$$

where $\mathrm{m}$ is the number of frequencies at which measurements are carried out, $\mathrm{n}$ is the number of adjusted parameters, and $\sigma\left(\varepsilon_{i}^{\prime}\right)$ and $\sigma\left(\varepsilon_{i}^{\prime \prime}\right)$ are the standard
deviations.

The full lines in Figs. 2,3, and 7 are computer plots of the type sketched above.

For a survey on dispersion equations and discussion of their features see ref. (1).

\section{DISCUSSION OF PERMITTIVITIES AND RELAXATION TIMES}

\section{General aspects}

The influence of the electrolyte on the dielectric properties of electrolyte solutions is evidenced by one or more of the following effects,

(a) decrease of the static permittivity of the solvent $\varepsilon_{S}(O)$ as a function of electrolyte concentration c yielding the "solvent permittivity" $\varepsilon_{s}(c)$ at concentration $c$ of electrolyte $\left(\varepsilon_{S}(c)=\varepsilon_{1}\right.$, eqs. (9), if no ion-pair relaxation exists, else $\left.\varepsilon_{s}(c)=\varepsilon_{2}\right)$,

(b) generation of electrolyte relaxation regions as a consecpuence of ion association yielding the "solution permittivity" $\varepsilon_{I P}(c)$. This is higher than $\varepsilon_{S}(c)\left(\varepsilon_{I P}(c)=\varepsilon_{1}\right.$, eqs. (9), if such a region exists),

(c) shift of the Solvent relaxation times $\tau_{\mathbf{s}}$ depending on electrolyte concentration $\mathrm{C}$,

(d) broadening of relaxation time distributions - increasing with electrolyte concentration $\mathrm{C}$,

(e) generation of relaxation times $\tau_{\text {IP }}$ - related to the appearance of electrolyte relaxation regions.

\section{Effects of electrolytes on solvent permittivity}

A characteristic feature of electrolyte solutions is the depression of the static permittivity $\varepsilon_{S}(c)$ of the solvent with regard to that, $\varepsilon_{s}(0)$, of the pure liquid. In high and moderate permittivity solvents where electrolyte relaxation regions are not observed, $\varepsilon_{s}(c)$ may be considered as the permittivity of the electrolyte solution. Examples of permittivity depression are 
given in Figs. $4 \mathrm{a}$ and $4 \mathrm{~b}$. The effect can be substantial as observed with a $1 \mathrm{M} \mathrm{LiNO}_{3}$ solution in $\mathrm{N}$-methylformamide. This produces a depression of permittivity from 181 (pure solvent) to 85 (solution) $(3,33)$.

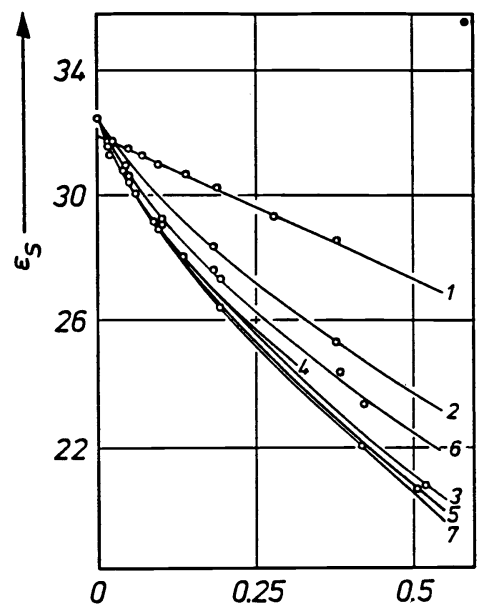

(a)

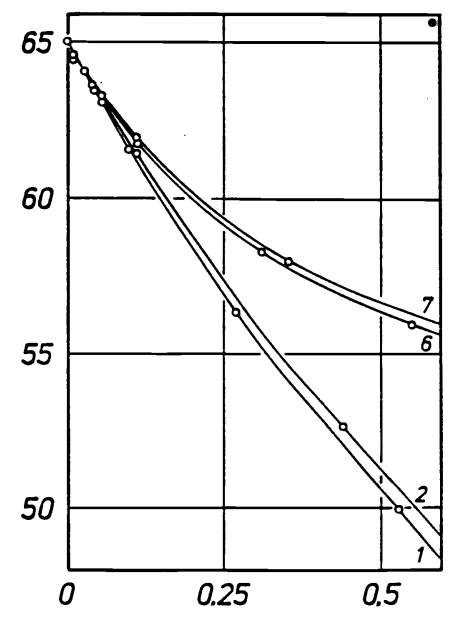

(b)

Fig. 4. Relative permittivities $\varepsilon_{\mathbf{S}}(\mathrm{c})$ of electrolyte solutions at $25^{\circ} \mathrm{C}$. (a) methanol solutions (36), (b) propilene carbonate solutions (12) of $1, \mathrm{Bu}_{4} \mathrm{NClO}_{4} ; 2, \mathrm{Bu}_{4} \mathrm{NI} ; 3, \mathrm{Bu}_{4} \mathrm{INBr} ; 4, \mathrm{NaCl}$; 5, NaBr; 6, $\mathrm{NaClO}_{4} ; 7$, NaI.

The comparison of Figs. $4 \mathrm{a}$ and $4 \mathrm{~b}$ shows completely different behaviour of methanol (amphiprotic hydroxylic) and propylene carbonate (aprotic protophobic) solutions by inverse sequences of the salts arranged according to increasing decrements.

Permittivity depression is always non-linear

$$
\varepsilon_{\mathbf{S}}(\mathrm{c})=\varepsilon_{\mathbf{S}}(0)-\delta \mathrm{c}+\beta \mathrm{C}^{\mathrm{n}} ; \mathrm{n}=2 \text { or } 3 / 2
$$

and specific for the electrolyte compound, see Figs. 4 and, e.g., aqueous solutions of alkali metal salts where permittivity depression increases in the sequences $\mathrm{Li}>\mathrm{Na}>\mathrm{K}>\mathrm{Rb}>\mathrm{Cs}$, and halides $>$ nitrates $(32,34,43)$.

The initial slope $\delta$ in eq. (11), the so-called dielectric decrement, is the quantity discussed in theoretical concepts of solvation $(2,5,35,45-48)$ and kinetic depolarization (49-52). It is obvious that precise measurements at low electrolyte concentrations are required for the determination of reliable values of $\delta$.

Several models have been proposed for the interpretation of dielectric decrements. These can roughly be classified in equilibrium approaches based on the concept of dielectric saturation, and dynamical theories based on kinetic depolarization.

Dielectric saturation is a consecuence of high field strength at the ionic surface yielding strong polarization and "irrotational bonding" of solvent molecules surrounding the ion. Solvation models based on this idea were proposed permitting a calculation of solvation numbers from dielectric decrements $(2,5,45-48)$; validity of the models is limited to dilute solutions. Actually two models are in current use; both models restrict molecule orientation to the next neighbours of the ion, the structure of the bulk solvent remains unchanged.

Pottel et al. $(45,46)$ use the semi-empirical Bruggeman relation for a calculation of the 'effective' volume fraction of the solvent. Comparison with its 'analytical' value yields solvation numbers $z_{p}$. $z_{p}$ equals 6.9 in water and 16.4 in methanol for a $0.25 \mathrm{M}$ solution of Licl (5). These numbers probably have little significance. Application of the model to low concentrations of $\mathrm{NaClO}_{4}$ and $\mathrm{Bu}_{4} \mathrm{NClO}_{4}$ solutions in methanol show a strong dependency of the calculated solvation numbers $z_{p}$ on concentration (36), thus $z_{p}=13.5$ at $0.01 \mathrm{~mol} \mathrm{dm}-3$ and $\mathrm{z}_{\mathrm{p}}=-10$ at $0.18 \mathrm{~mol} \mathrm{dm}-3$ for $\mathrm{Bu}_{4} \mathrm{NClO}_{4}$ solutions. 
Lestrade et al. $(2,47)$ use the Kirkwood-Fröhlich equation where the Kirkwood $g$ parameter is assumed to be independent of electrolyte concentration, ( $\partial \mathrm{g} / \partial \mathrm{c})$ $=0$, to calculate solvation numbers $\mathrm{z}_{\mathrm{L}}$ via the number of molecules per unit volume, qc, causing the limiting slope of permittivity depression $\delta, \delta=$ $-\lim \left(\partial \varepsilon_{s}(c) / \partial t\right)$

$\mathrm{C} \rightarrow \mathrm{O}$

$$
\mathrm{z}_{\mathrm{L}}=\left(\rho-\rho_{0}+q \mathrm{q}\right) / \mathrm{c} ; \mathrm{q}=\delta \frac{\rho_{0}}{\varepsilon_{\mathrm{S}}(0)} \frac{2 \varepsilon_{\mathrm{S}}^{2}(0)+\varepsilon_{\infty}^{2}(0)}{\left(\varepsilon_{\mathrm{S}}(0)-\varepsilon_{\infty}(0)\right)\left(2 \varepsilon_{\mathrm{S}}(0)+\varepsilon_{\infty}(0)\right)}
$$

In eqs. (12) $\rho$ is the number density of solvent in the solution of electrolyte concentration $c, \rho_{0}$ is the number density of the pure solvent.

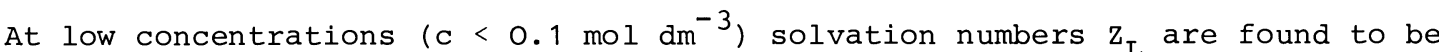
independent of concentration. Examples are given in Table 3 for methanol and propylene carbonate solutions.

TABLE 3. Solvation numbers from dielectric decrements

\begin{tabular}{lccccc}
\hline salt & \multicolumn{2}{c}{ propylene } & carbonate & (12) & \multicolumn{2}{c}{ methanol } & (36) \\
\hline & $\mathrm{z}_{\mathrm{L}}$ & $\mathrm{z}_{\mathrm{HO}}$ & $\mathrm{z}_{\mathrm{P}}$ & $\mathrm{z}_{\mathrm{L}}$ & $\mathrm{z}_{\mathrm{HCW}}$ \\
$\mathrm{NaBr}$ & - & - & 20.1 & 32.0 & 3.9 \\
$\mathrm{NaI}$ & 5.5 & 3.9 & 18.6 & 29.7 & 1.5 \\
$\mathrm{NaClO}$ & 6.0 & 4.4 & 14.1 & 20.1 & 3.2 \\
$\mathrm{Bu}_{4} \mathrm{NBr}$ & - & - & 3.2 & 20.4 & 5.8 \\
$\mathrm{Bu}_{4} \mathrm{NI}$ & 3.8 & 2.3 & -2.0 & 3.5 & $\mathrm{O}$ \\
$\mathrm{Bu}_{4} \mathrm{NClO}_{4}$ & 4.3 & 2.8 & -9.0 & 3.2 & $\mathrm{O}$
\end{tabular}

Solvation numbers $\mathrm{z}_{\mathrm{L}}$ (Lestrade et al. (2)); $\mathrm{z}_{\mathrm{p}}$ (Pottel et al. (46)); $\mathrm{z}_{\mathrm{HO}}$ and $\mathrm{z}_{\mathrm{HCW}}$ are $\mathrm{z}_{\mathrm{L}}$ numbers corrected for kinetic depolarization with the continuum (eq. (13b)) or microscopic (eq. (13c)) model; $Z_{\mathrm{L}}, \mathrm{Z}_{\mathrm{HO}}$ independent of concentration; $\mathrm{Z}_{\mathrm{P}}$, $\mathrm{Z}_{\mathrm{HCW}}$ at $0.1 \mathrm{~mol} \mathrm{dm}^{-3}$.

Solvation numbers of propylene carbonate solutions (12) seem to be quite reasonable, similar values for $z_{\mathrm{I}}$ are also found for other aprotic solvent systems (2). Solutions of protic solvents, methanol (Table 3), ethanol (47) and also water (53), show very large $z_{\text {f }}$ attributed by Badiali (47) to solvation of the cations by alcohol multimers. This is in contrast to the results of Jorgensen et al. (54) who find, from computer simulations, a tight fitting first solvation shell of six methanol molecules around the sodium ion but almost no interaction at larger distances.

The theories of kinetic depolarization (49-52) link the decrease of static permittivity $\Delta \varepsilon, \Delta \varepsilon=\varepsilon_{\mathbf{S}}(\mathrm{O})-\varepsilon_{\mathbf{S}}(\mathrm{c})$, to ionic motions. Hubbard, Colonomos and Wolynes (51) showed that the corrected original continuum theory predicts proportionality of $\Delta \varepsilon$ and specific conductance

$$
\Delta \varepsilon=\xi \cdot \kappa ; \quad \xi=p \frac{\varepsilon_{S}(0)-\varepsilon_{\infty}(0)}{\varepsilon_{S}(0)} \cdot \frac{\tau_{S}(0)}{\varepsilon_{0}}
$$

The constant of proportionality $\xi$ is solely determined by the dielectric data of the solvent and the friction factor $p$ characterizing hydrodynamic boundary conditions; $p=1$ for sticking, $p=2 / 3$ for slipping movement of the ions.

Linear relationships $\Delta \varepsilon$ us.K are usually found, as seen in Fig. 5. Ranges of linearity may cover large concentration ranges, despite the theoretical limitations of eqs. (13) to low concentrations, e.g. up to $4 \mathrm{~mol} \mathrm{dm}^{-3}$ for aqueous solutions of $\mathrm{KCl}$ (53). Only NaI solutions in DMSO seem to be an exception (11) to this.

Eqs. $(13 a, b)$ are fulfilled quantitatively for solutions of water and $\mathrm{SO}_{3}$ in concentrated sulfuric acid (55). In general, however, the experimental de-

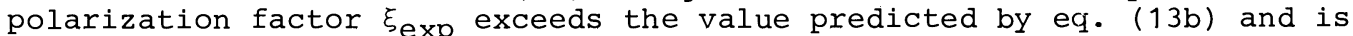
specific for solvent and solute, see Fig. (5), in contrast to the theory.

To overcome this difficulty corrected solvation numbers were calculatea $(5,6$, $12,35,45)$ based on the superposition of kinetic depolarization and dielectric saturation. The result is reasonable for aprotic (12) but not convincing for protic solvents. Methanol solutions yield concentration dependent solvation 
numbers at low concentrations and the results are not self-consistent for a series of salts in this solvent. For example, the bromide ion would have a solvation number of zero in $\mathrm{NaBr}$ and 5.8 in $\mathrm{Bu}_{4} \mathrm{NBr}$ (36). Another solution of the problem is given by Felderhof (52) who takes into account the proper volume of solutes.

The microscopic model of Hubbard, Colonomos and Wolynes, also published in ref. (51), permits the calculation of depolarization factors from reduced ionic decrements $A_{+}$and $A_{-}$and transference numbers $t_{+}$and $t_{-}(+$, cation; -, anion).

$$
\xi=\left(\tau_{s}(0) / \varepsilon_{0}\right)\left(t_{+} A_{+}+t_{-} A_{-}\right)
$$

Up to now reduced ionic decrements are available only for water and methanol as a function of ionic radii. A consistent series of decrements is found for the methanol solutions in Table 3 if the effective radius of $\mathrm{Na}^{+}$is set to the sum of the ion radius and the diameter of an orientated methanol molecule, reff $=a\left(\mathrm{Na}^{+}\right)+\mathrm{s}, \mathrm{c}$. the chemical model of methanol solutions (44).

A unified theoretical treatment is desirable for a better understanding of the dielectric permittivity of electrolyte solutions which takes into account both static and dynamic effects. An interesting approach to this problem is provided by Patey et al. (56-58). They used HNC theory in a way originally suggested by Friedman (59). Up to now calculations have been performed only for a waterlike model solvent.

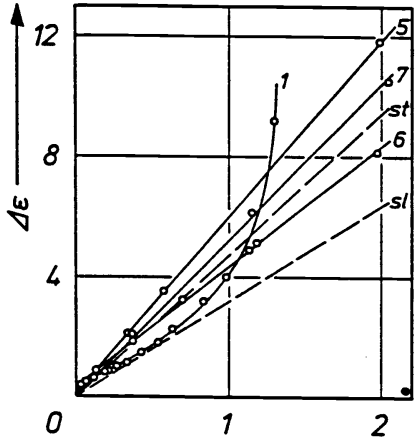

(a)

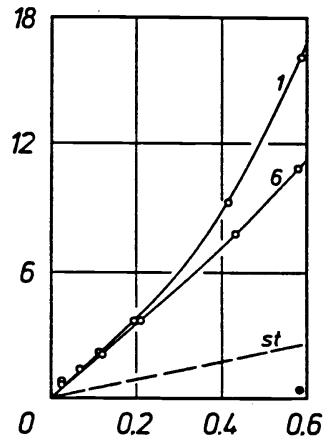

(b)

Fig. 5. Dielectric decrements, $\Delta \varepsilon=\varepsilon_{\text {(O) }}-\varepsilon_{\mathrm{S}}$ (c), of (a) methanol (36) and (b) propylene carbonate ( 12 ) solutions at $25^{\circ} \mathrm{C}$ of $1, \mathrm{Bu}_{4} \mathrm{NClO}_{4} ; 5, \mathrm{NaBr} ; 6, \mathrm{NaClO}_{4} ; 7, \mathrm{NaI}$. The broken lines show the calculated decrements, eqs. $(13 a, b)$, for stiction (st, $\mathrm{p}=1$ ) and sliding friction ( $1, \mathrm{p}=2 / 3$ ) of the ions.

The preceding discussion concerns the decrease of solvent permittivity as the result of ion-solvent interaction. Another effect is discussed in the lierature. Debye and Falkenhagen (60) predicted the disrersion of conductance caused by the relaxation of the ionic cloud around a reference ion. Consequently, the separation of permittivity and conductance according to ecis. (7) should result in an increase of the static permittivity of the electrolyte solution. With reference to the work of van Beek and Mandel (8) several authors take this effect into account when interpreting experimental results $(7,57)$. However, no significant contribution of the Debye-Falkenhagen effect is detectable within the experimental accuracy of complex permittivity measurements $(6,11$, $29,36,53)$, see also Cavell's argument (61). Nor do conductance measurements yield conclusive results in the expected frequency range (62). Hence, the splitting of the total complex permittivity $\varepsilon_{\text {tot }}^{\prime}$ as in eqs. (7) is justified.

For all systems studied up to now the high frequency permittivity surpasses $n_{1 R}$, (n $n_{\text {IR }}$ refractive index from IR measurements) reflecting contributions from short time motions. Suffice it to say that $\varepsilon_{\infty}$ is independent of the nature and the concentration ( $c$ f. Fig. 2a) of methanol electrolyte solutions within limits of error, $c f$. (36), in contrast to solutions of inert solutes like $\mathrm{MeOH}^{-\mathrm{CCl}_{4}}{ }^{-}$ mixtures where a minimum in $\varepsilon_{\infty}$ is found (63). 


\section{Effects of electrolytes on solvent relaxation times}

Dielectric relaxation times and their continuous or discontinuous distributions are an important source of information on dynamic processes in solution. A major drawback to an exhaustive determination of relaxation modes is the limited frequency coverage of the experimental equipment. It is not unusual that the description of a system appropriate for a given data set has to be corrected if the frequency range is expanded. For methanol a cole-Cole plot is optimal for data below $12 \mathrm{GHz}$. Inclusion of data up to $18 \mathrm{GHz}$ or more into the data analysis requires the superposition of two Debye processes, $i . e$. the discussion of two distinctly different relaxation times instead of a continuous relaxation time distribution around a critical relaxation time (36). However, the main relaxation times of the solvent, $\tau_{s}(0)$ or $\tau_{1}(0)$, do not change significantly with the frequency range used for their calculation and the appearance of high frequency modes, cf. Table 1, and their relative shifts with electrolyte concentration remain almost unaffected.

The dielectric relaxation time is a collective quantity related to the step response function of polarization $\mathrm{F}_{\mathrm{p}}$, eq. (4). Direct access to molecular motions is given via the autocorrelation function of the molecular dipole moment $\vec{\mu}(t)$

$$
\phi(t)=\langle\vec{\mu}(0) \cdot \vec{\mu}(t)\rangle /\langle\vec{\mu}(0) \cdot \vec{\mu}(0)\rangle
$$

or the corresponding microscopic (or molecular) relaxation time $\tau_{s}^{\prime} ; \phi(t)$ can be related to $F_{p}$ with the help of a complicated procedure, due to the long range dipolar interaction forces of the molecules. For details of calculation reference is made to the literature $(1,64)$. With simple liquids the powlesGlarum relation $(65,66)$

$$
\tau_{s}^{\prime}=\frac{2 \varepsilon_{S}+\varepsilon_{\infty}}{3 \varepsilon_{s}} \cdot \frac{\tau_{s}}{g}
$$

may be used where $g$ is the Kirkwood correlation factor; $\tau$ 's often is correlated with dynamic viscosity $n$ via the generalized Stokes-Einstein-Debye equation (67)

$$
\tau_{s}^{\prime}=\frac{3 V \eta}{k T} f_{s t} \cdot C+\tau_{s}^{\prime o}
$$

where $\mathrm{V}$ is the molecular volume of the relaxing compound, $f$ is a stick parameter depending on molecular shape, $C$ is an experimental partameter coupling microscopic and macroscopic viscosity, and $\tau_{\mathrm{S}}^{\prime} \mathrm{O}$ is the experimentally aetermined intercept with the $\tau^{\text {-axis }}(n=0)$, sometimes referred to as the free rotor correlation time. A relationship of the type of eq. (16) was verified in $\mathrm{Bu}_{4} \mathrm{NI}$ and $\mathrm{Bu}_{4} \mathrm{NClO}_{4}$ solutions of propilene carbonate (12).

Associating liquids usually show complex relaxation behaviour. Their main dispersion step (generally that with the longest relaxation time) corresponds to the rearrangement of the liquid structure (1). A decrease or increase of the main relaxation time with electrolyte concentration may be interpreted as a structure making or breaking effect induced by the solute.

Solutions in methanol $(63,69)$ and ethanol $(69)$ were the first non-aqueous systems studied. Older measurements of electrolyte solutions in these solvents $(33,47,68-70)$ as well as recent time domain results (6) were analysed with a single Debye process neglecting high frequency contributions to orientation polarization. No significant changes of $\tau_{\mathbf{s}}$ were found in these investigations on the addition of salts, in contrast to recent research.

For methanol the existence of a high frequency process with a time constant $\tau_{2}\left(\tau_{2} \sim 5 \mathrm{ps}\right)$ beyond the main relaxation time $\tau_{\mathbf{s}}\left(\tau_{\mathbf{s}}=\tau_{1} \sim 50 \mathrm{ps}\right)$ is clearly established $(5,36)$ although the origin of $\tau_{2}$ remains a matter for discussion (63). For electrolyte solutions the data spread is too larce for a detailed discussion of $\tau_{2}$, but an increase in $\tau_{2}$ with electrolyte concentration is always observed (36).

Kaatze et al. (6) fitted their data by superimposing two exponentials. They did not find a change in the low frequency relaxation time $\tau_{1}$ for $1 \mathrm{M}$ solutions of NaBr, NaI and KI, but complex behaviour was observed for LiCl with minima and maxima. 

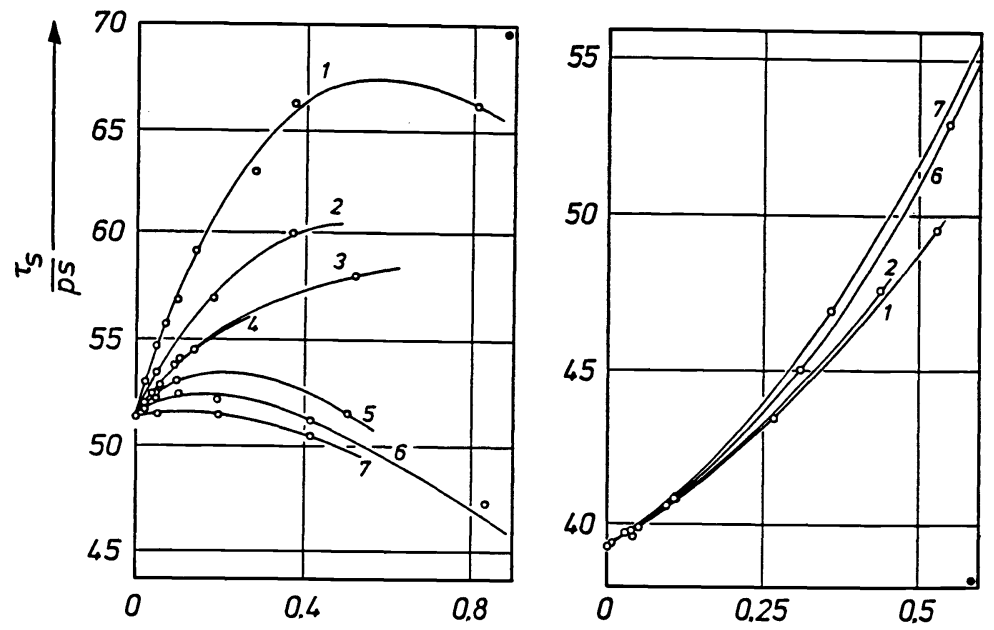

(a)

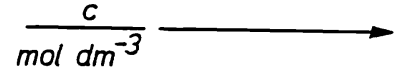

(b)

Fig. 6. Main relaxation time $\tau_{S}(\mathrm{c})$ of the solvent at $25^{\circ} \mathrm{C}$ in

(a) methanol solutions (36), (b) propylene carbonate solutions

(12) of 1, $\mathrm{Bu}_{4} \mathrm{NClO}_{4} ; 2, \mathrm{Bu}_{4} \mathrm{NI} ; 3, \mathrm{Bu}_{4} \mathrm{NBr} ; 4, \mathrm{NaCl} ; 5, \mathrm{NaBr}$;

$6, \mathrm{NaClO}_{4} ; 7, \mathrm{NaI}$.

Our methanol relaxation times (36), Fig. 6a, result from such an analysis. A striking feature is the salt specificity observed and the maximum of $\tau$, (see Fig. 6a) suggesting the competition between structure making and breaking effects. It is interesting to note that for tetraalkylammonium salts the order of relaxation times parallels that obtained for ion sizes whereas for the sodium salts the order is the same as the sequence of molar refractions. The domination of the structure breaking effects for the alkali metal salts can be understood by strong cation solvation. A maximum of $\tau_{1}$ was also found for 1-propanol solutions (10).

$\mathrm{N}$-methylformamide as a protic solvent in its solutions of $\mathrm{LiNO}_{3}$ (32) and NaI (11) shows decreasing relaxation times combined with considerable broadening of the relaxation time distribution for LiNO $_{3}$. This and the very large decrease of static permittivity is in accord with the destruction by the electrolyte of the linear molecular associates of the pure solvent (3,33). The relaxation time of formamide, a solvent with $\mathrm{H}$-bonded network similar to water, decreases on addition of NaI (11), but increases with LiNO $_{3}$ (33). This is in contrast to water where increasing relaxation times are reported only for tetraalkylammonium bromides (45) and alkali metal fluorides (43). At present information on amides is too limited to allow discussion. However, these solvents with their widely ranging structural properties are of interest in fundamental research and deserve more attention.

Among the aprotic dipolar solvents of Table 1 only propylene carbonate has been thoroughly re-investigated $(12,14)$. The other non-H-bonded solvents were treated with the help of single relaxation time dispersion functions. For all aprotic solvents relaxation times increase with electrolyte concentrations, and Fig. 6b shows the solute specificity of relaxation times for propylene carbonate solutions (12); again the sequence of salts is inverted when comparea to methanol solutions.

The low permittivity and viscosity solvents of Table 2 exhibit no change in relaxation time, possibly due to the spanning of too short a frequency range. For these solvents the observed relaxation time is not always due to the rearrangement of the whole molecule (14).

Information on relaxation times from rate processes are characterized by activation parameters (23). Unfortunately, temperature dependence data are lacking and the discussion of this important aspect is not possible at present.

\section{Ion-pair relaxation}

Electrolytes in low permittivity solvents $(\varepsilon<10)$ are highly associated to ion pairs and higher aggregates, see refs. $(2,4,44$ and literature quoted therein). Electrolyte solutions of this type have considerable technological 
interest (44), for instance lithium salts with electron donor solvents are commonly used in high energy batteries.

Due to the high concentration in these solutions of ion pairs, stable within the time scale of the dielectric experiments, an additional dispersion region is found at the low frequency side of the dielectric spectrum attributable to the motions of ion pairs or higher aggregates. Ion-pair relaxation of trialkyland tetraalkylammonium salts in apolar and low permittivity solvents was extensively studied by Cavell et al. $(71,72)$ in the 1970's. Electrolyte solutions relevant for lithium batteries were investigated by Lestrade et al. $(13,48)$, and Petrucci et al. $(15-21)$.

The contribution of ion-pair relaxation to orientational polarization can usually be described by a Debye equation with single relaxation time (see Fig. 3) or by a Cole-Davidson equation with a large distribution parameter (2). The dispersion amplitude of the electrolyte relaxation region compensates partly, or sometimes even overcompensates, for the dielectric decrement of the solvent (see Fig. 3) so that addition of electrolyte would cause an increase in solution permittivity.

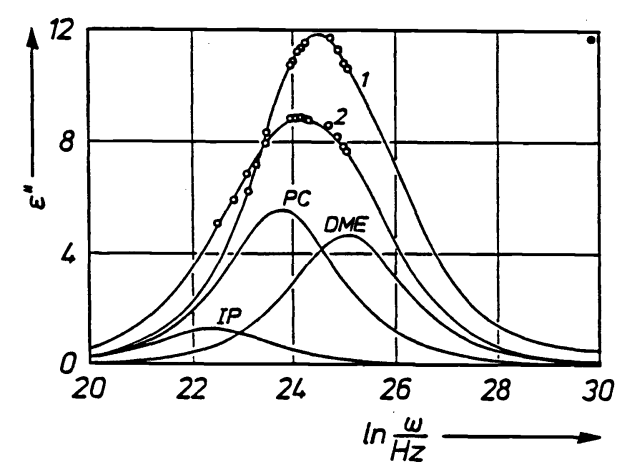

Fig. 7. Absorption curves of the system propylene carbonate-1.2-dimethoxyethane$0.43 \mathrm{M} \mathrm{NaClO}_{4}$ at $25^{\circ} \mathrm{C}(14)$. Curve 1 , pure solvent system $\left(x_{\mathrm{PC}}=0.42\right)$; curve 2 , electrolyte solution; PC, DME, IP, bands obtained by analysis of curve 2 .

Absorption àiagrams, $\varepsilon^{\prime \prime}=\mathrm{f}(\ln \omega)$, yield ion-pair bands by band analysis and dispersion diagrams, $\varepsilon^{\prime}=f(\ln \omega)$, confirm these results by the corresponding dispersion steps. Fig. 7 illustrates the change in absorption by the aadition of $\mathrm{NaClO}_{4}(0.5 \mathrm{II})$ to a solvent mixture of propylene carbonate and 1.2 -dimethoxyethane $\left(\mathrm{x}_{\mathrm{PC}}=0.42\right)$ and the decomposition of the absorption curve of the $0.5 \mathrm{M}$ solution into bands for DME, PC, and IP (14). With regard to the absorption curve of the pure solvents (curve 1) the curve of the $0.5 \mathrm{M}$ electrolyte solution (curve 2) is shifted to lower frequencies due to the increase of relaxation times of the solvent molecules. It is unsymmetrically broadened on its low frequency side on the addition of salt with the appearance of the ion-pair band. Further addition of DME causes the formation of a shoulder; this situation is given for the solution $\left(x_{P C}=0.20\right)$ of which the Cole-Cole diagram is shown in Fig. 3.

The representation of an ion-pair relaxation region by a Debye-process with a single relaxation time ${ }^{\tau} I P$ is compatible with the model of a rigid dipole for the ion pair. Analysis of the dispersion amplitude $\left(\varepsilon_{I_{P}}(\mathrm{c})-\varepsilon_{\mathrm{S}}(\mathrm{c})\right.$ ) can be achieved with a modified Onsager-Böttcher equation $(7 \uparrow, 72)$.

$$
\frac{\left(2 \varepsilon_{I P}(c)+1\right)\left(\varepsilon_{I P}(c)-\varepsilon_{S}(c)\right)}{3 \varepsilon_{I P}(c)}=\frac{N_{A} c_{I P} \cdot 10^{-3}}{\left(1-\alpha_{I P} f\right)^{2}} \cdot \frac{\mu_{I P}^{2}}{3 k T \varepsilon_{O}}
$$

In eq. (17) $c_{I P}$ is the concentration of ion pairs [mol $\mathrm{dm}^{-3}$ ], $\mu_{I P}$ and $\alpha_{\text {IP }}$ are the dipole moment and the polarizability of the ion pair, $f$ is the factor of the reaction field; the other symbols have their usual meaning. Deviations from linearity of the plot of the left hand side expression us. ion-pair concentration have been attributed to the formation of polar (17) or apolar (21) quadruples. The latter associates are supposed to be dominant in solutions of $\mathrm{LiBF}_{4}$ in dimethoxymethane where no solute dispersion was found (19).

The origin of the solute dispersion steps for $\mathrm{Ca}\left(\mathrm{NO}_{3}\right)_{2} \cdot 4 \mathrm{H}_{2} \mathrm{O}$ in acetone (22) and for $\mathrm{ZnBr}_{2}$ in propylene carbonate (12) is more complex and requires further study using different approaches for a greater clarification.

Models taking into account rotational and translational modes of ion-pair motion (72-74) to explain deviations from Debye behaviour were criticized by 
Petrucci et al. (18).

Ion-pair relaxation in a high permittivity solvent is observed in the case of aqueous 2-2 electrolytes $(75,76)$. The solvent-separated ion pairs of $\mathrm{MgSO}_{4}$ and $\mathrm{CdSO}_{4}$ were identified as the relaxing species from both the dispersion amplitude and the relaxation time (76). Also, an estimation of the rate constant of ion-pair formation was possible from the concentration dependency of the ion-pair relaxation time $\tau_{\text {IP }}(53)$.

\section{REFERENCES}

1. C. F. J. Böttcher and P. Bordewijk, Theory of Electric Polarization, Elsevier, Amsterdam (1978).

2. J.-C. Lestrade, J. P. Badiali and H. Cachet, Dielectric and Related Molecular Properties, Ed. by M. Davies, Vol. 2, 106-150, The Chemical Society, London (1975).

3. J. Barthel, Pure Appl. Chem. 51, 2093-2124 (1979).

4. J. Barthel, Ionen in nichtwäßrigen Lösungen, Steinkopff Darmstadt (1976).

5. U. Kaatze, D. Adolph, D. Gottlob, and R. Pottel, Ber. Bunsenges. Phys. Chem. $34,1198-1203$ (1980).

6. P. Winsor IV and R. H. Cole, J. Phys. Chem. 86, 2491-2494 (1982).

7. J. B. Hubbard, L. Onsager, W. M. van Beek, and M. Mandel, Proc. Natl. Acad. Sci. USA 74, 401-404 (1977).

8. W. M. van Beek and M. Mandel, J. Chem. Soc. Faraday Trans. I 74, 2339-2351 (1978).

9. R. Pottel, Ber. Bunsenges. Phys. Chem. 75, 286-294 (1971).

10. B. Gestblom, S. C. Mehrotra, and J. Sjöblom, J. Solution Chem. 15, 55-68 (1986).

11. P. Vinsor IV and R. H. Cole, J. Phys. Chem. 86, 2486-2490 (1982).

12. J. Barthel and F. Feuerlein, J. Solution Chem. 13, 393-417 (1984).

13. H. Cachet, M. Fekir, and J.-C. Lestrade, Can. J. Chem. 59, 1051-1060 (1981).

14. J. Barthel and F. Feuerlein, submitted for publication.

15. H. Farber, D. E. Irish, and S. Petrucci, J. Phys. Chem. 87, 3515-3520 (1983).

16. H. E. Maaser, M. Delsignore, M. Newstein, and S. Petrucci, J. Phys. Chem. 88, 5100-5107 (1984).

17. H. Farber and S. Petrucci, J. Phys. Chem. 85, 2987-2992 (1981).

18. D. Saar, J. Brauner, H. Farber, and S. Petrucci, J. Phys. Chem. 84, 341349 (1980).

19. M. Delsignore, H. Farber, and S. Petrucci, J. Phys. Chem. 90, 66-72 (1986).

20. M. Delsignore, H. E. Maaser, and S. Petrucci, J. Phys. Chem. 88 , 24052411 (1984).

21. M. Delsignore, H. Farber, and S. Petrucci, J. Phys. Chem. 89, 4968-4973 (1985).

22. B. Gestblom and J. Sjöblom, Chem. Phys. Lett. 122, 553-566 (1985).

23. N. E. Hill, W. E. Vaughan, A. H. Price, and M. Davies, Dielectric Properties and Molecular Behaviour, Van Nostrand Reinhold Co., London (1969).

24. $\mathrm{H}$ igh Frequency Dielectric Measurements, ed. by J. Chamberlain and G. W. Chantry, I. P. C. Science and Technology Press Ltd. Guilford (1973).

25. E. H. Grant, R. J. Sheppard, and G. P. South, Dielectric Behaviour of Biological Molecules in Solution, Ch. 3, Oxford University Press, Oxford (1978).

26. U. Kaatze and K. Giese, J. Phys. E: Sci. Instrum. 13, 133-141 (1980).

27. R. H. Cole, Ann. Rev. Phys. Chem. 28, 283-300 (1977).

28. R. H. Cole and P. Winsor IV, in: Fourier, Hadamard and Hilbert Transforms in Chemistry, Ed. by A. G. Marshall, 183-206, Plenum, New York (1982).

29. P. Winsor IV and R. H. Cole, J. Phys. Chem. 39, 3775-3776 (1985).

30. T. J. Buchanan, Proc. IEE Part III 99, 61-66 (1952).

31. J. Krüger, E. Schollmeyer, and J. Barthel, Z. Naturforsch. 30a, 1476-1480 (1975).

32. J. Barthel, F. Schmithals, and H. Behret, Z. Phys. Chem. NF 71, 115-131 (1970).

33. J. Barthel, H. Behret, and F. Schmithals, Ber. Bunsenges. Phys. Chem. 75 , 305-309 (1971).

34. H. Behret, F. Schmithals, and J. Barthel, Z. Phys. Chem. NF 96, 73-83 (1975) .

35. U. Kaatze and R. Pottel, Z. Phys. Chem. NF 141, 1-13 (1984).

36. J. Barthel, K. Bachhuber, R. Buchner, and B. Kaukal, in preparation.

37. E. A. S. Cavell, J. Chem. Soc. Faraday Trans. II 70, 78-84 (1974).

38. P. Debye, Polar Molecules (reprint of the 1st edition), Dover Publications. 
39. K. S. Cole and R. H. Cole, J. Chem. Phys. 9, 341-351 (1941).

40. D. W. Davidson and R. H. Cole, J. Chem. Phy̆s. 18, 1417 (1950).

41. S. Havriliak and S. Negami, J. Polym. Sci. C14, 99-103 (1966).

42. H. Fröhlich, Theory of Dielectrics, 2nd ed., Oxford University Press (1958).

43. J. Barthel, J. Krüger, and E. Schollmeyer, Z. Phys. Chem. NF 104, 59-72 (1977).

44. J. Barthel, H.-J. Gores, G. Schmeer, and R. Wachter, Topics in Current Chemistry, Ed. by F. L. Boschke, Springer, Heidelberg, 111, 33-144(1983).

45. U. Kaatze, Z. Phys. Chem. NF 135, 51-75 (1983).

46. K. Giese, U. Kaatze, and R. Pottel, J. Phys. Chem. 74, 3718-3725 (1970).

47. J.-P. Badiali, H. Cachet, and J.-C. Lestrade, J. Chim. Phys. 25, 1350-1359 (1967).

48. J.-P. Badiali, H. Cachet, and J.-C. Lestrade, Pure Appl. Chem. 53, 13831399 (1981).

49. J. B. Hubbard and L. Onsager, J. Chem. Phys. 67, 4850-4857 (1977).

50. J. B. Hubbard, J. Chem. Phys. 68, 1649-1664 $(1978)$.

51. J. B. Hubbard, P. Colonomos, and P. G. Wolynes, J. Chem. Phys. 71, 26522661 (1979).

52. B. U. Felderhof, Mol. Phys. 51, 801-811 (1984).

53. J. Barthel, R. Buchner, and $\mathrm{K}$. Popp, in preparation.

54. W. L. Jorgensen, B. Bigot, and J. Chandrasekhar, J. Amer. Chem. Soc. 104, 4584-4591 (1982).

55. D. G. Hall and R. H. Cole, J. Phys. Chem. 35, 1065-1069 (1981).

56. G. N. Patey and S. L. Carnie, J. Chem. Phys. 78, 5183-5190 (1983).

57. P. G. Kusalik and G. N. Patey, J. Chem. Phys. 79, 4463-4474 (1983).

58. G. N. Patey and F. Garisto, Chem. Phys. Lett. 117, 457-460 (1985).

59. H. L. Friedman, J. Chem. Phys. 76, 1092-1105 (1982) .

60. H. Falkenhagen, Therie der Elektrolyte, S. Hirzel Verlag, Leipzig (1971).

61. E. A. S. Cavel1, J. Chem. Soc. Faraday Trans. II 78, 537-548 (1982).

62. J. Barthel and H. -J. Wittrann, in preparation.

63. J. Barthel and R. Buchner, in preparation.

64. P. Madden and D. Kivelson, Adv. Chem. Phys. 56, 467-566 (1984).

65. J. G. Powles, J. Chem. Phys. $21,633-637$ (1953).

66. S. H. Glarum, J. Chem. Phys. 33, 1371-1375 (1960).

67. J. C. Dote, D. Kivelson, and R. N. Schwartz, J. Phys. Chem. 85, 2169-2180 (1981).

68. J. A. Lane and J. A. Saxton, Proc. Roy. Soc. A214, 531-545 (1952).

69. J. B. Hasted and G. W. Roderick, J. Chem. Phys. 29, 17-26 (1958).

70. H. Cachet, J. Epelboin, and J.-C. Lestrade, Electrochim. Acta 11, 17591769 (1966).

71. E. A. S. Cavell, P. C. Knight, and M. A. Sheikh, J. Chem. Soc. Faraday Trans. 67, 2225-2233 (1971).

72. E. A. S. Cavell and P. C. Knight, J. Chem. Soc. Faraday Trans. II 68, 765-775 (1972).

73. H. Farber and S. Petrucci, J. Phys. Chem. 79, 1221-1227 (1975).

74. H. Cachet, A. Cyrot, M. Fekîr, and J.-C. Lestrade, J. Phys. Chem. 83 , 2419-2429 (1979).

75. R. Pottel, Ber. Bunsenges. Phys. Chem. 69, 363-378 (1965).

76. J. Barthel, R. Buchner, and H.-J. Wittmann, Z. Phys. Chem. NF 139, 23-37 $(1984)$.

77. P. R. Bevington, Data Reduction and Error Analysis for the Physical Sciences, Mc Graw Hill, New York (1969).

78. R. M. Fuoss and J. G. Kirkwood, J. Amer. Chem. Soc. 63, 385-394 (1941).

79. J. Barthel and H. Steger, unpublished data. 